\title{
Bone marrow mesenchymal cell transplantation for heart diseases: mechanisms and applications
}

\author{
Chun-Yan Zhou ${ }^{1}$ \\ ${ }^{1}$ School of Basic Medical Sciences, Peking University, Beijing, China
}

Current therapeutic strategies for the treatment of end-stage cardiac failure are limited. As the number of patients with refractory myocardial ischemia and congestive heart failure is rapidly rising there is urgent need for development of novel therapeutic approaches. Recent experimental studies based on innovative hypotheses utilizing cell therapy for damaged myocardium are promising. A number of cell types, including skeletal myoblasts, fetal cardiomyocytes, smooth muscle cells, embryonic stem cells and bone marrow mesenchymal cells (MSCs) have been tested for this purpose. Most evidence shows that MSCs might be one of the promising candidates as a therapeutic tool for the treatment of myocardial infarction and end-stage cardiac failure. Using a rat model with acute infarct heart generated by ligation of left anterior descending coronary artery, the mechanisms of transplanted MSCs on myocardial function recovery were investigated. The results confirmed that transplantation of MSCs improved cardiac function as assessed by measuring left ventricle ejection fraction and fraction of shortening based on Echocardiography. Several factors might contribute to this functional improvement. First, transplanted MSCs expressed the specific markers for cardiomyocytes and endothelial cells 14 days after transplantation, indicating that these cells gained new phenotypes. Second. Transplantation of MSCs upregulated endogenous VEGF and HO-1 expression in an early stage of heart infarction, thus enhanced angiogenesis and myocardial protection. Third, MSCs transplantation inhibited cardiomyocytes apoptosis by regulating the expressions of bcl-2 and bax. Fourth, MSC transplantation led to dynamic changes in the collagen network through regulation of MMP2/TIMP1 and consequently interrupted the progress of adverse left ventricle's remodeling. Fifth, some cytokines or growth factors either secreted by transplanted cells or endogenous cells might contribute to the improvement of damaged heart function.

Pig models with acute infarct heart generated by ligation of left anterior descending coronary artery or chronic heart infarction created by using ameroid constrictors were used to evaluate the safety and the procedures of MSCs transplantation for the treatment of heart infarction. It was proved to be effective in restoring myocardial function that transplanting cells should be more than 108 cells. The induced arrhythmia was less than the control group (pigs without MSCs transplantation). The transplantation time after infraction and injection sites were also optimized. No restenosis was observed. Based on the data from animal research, we carried out a clinical trial of autologous bone marrow cells treatment for chronic heart failure. A total of 46 patients were entered and the follow-up observation was continued for 36 months. Cell transplantation improved heart function about $5 \%$ over the normal treatment, improved cardiomyocyte perfusion and metabolism. However, we also observed that restenosis was increased with application of bare metal stemt in cell transplantation group compared with normal treatment group.

Keywords: bone marrow mesenchymal cells, cardiomyocyte differentiation, transplantation

Cell Research (2008) 18:s119. doi: 10.1038/cr.2008.209; published online 4 August 2008

Correspondence: Chun-Yan Zhou

E-mail: chunyanzhou@bjmu.edu.cn

Chun-Yan Zhou, MD, PhD, was trained as a medical doctor in Harbin Medical University, received her Master degree from the Chinese Academy of Preventive Medical Sciences and a PhD from University of Cambridge. Dr Zhou is a current full Professor of Peking University Health Science Centre, Associate Dean in School of Basic Medical Sciences. She has been elected as vice Director of the Chinese Society of Medical Biochemistry and Molecular Biology (2007-2011). She is also a member of the fifth editorial board, Chinese Journal of Biochemistry and Molecular Biology. Dr Zhou's research focuses on the gene expression regulation during the stem cell differentiation, particularly the proliferation and differentiation of bone marrow stem cells in damaged heart.

For more details: http://medicine.bjmu.edu.cn//20041027/article/200604/10778.htm. 